\title{
The Use of Placebo and the Right of Autonomy
}

Kam-Yuen Cheng *

Centennial College, 3 Wah Lam Path, Pokfulam, Hong Kong

*Corresponding author: Kam-Yuen Cheng, Centennial College, 3 Wah Lam Path, Pokfulam, Hong Kong, Tel: 852 3762 624; E-mail: kycheng@hkuspace.hku.hk

Rec date: Dec 09, 2014, Acc date: Jan 12, 2015, Pub date: Jan 15, 2015

Copyright: (C) 2015 Cheng K. This is an open-access article distributed under the terms of the Creative Commons Attribution License, which permits unrestricted use, distribution, and reproduction in any medium, provided the original author and source are credited.

\begin{abstract}
In the clinical trial context, given that placebo-controlled trials do not subject participants to serious or irreversible harm, placebo use is ethically justified because the participation is autonomous. In the clinical practice context, assuming that the open use of placebos is ineffective, placebos can be used deceptively without violating patients' right of autonomy and therefore can also be ethically justified.
\end{abstract}

Keywords: Placebo; Autonomy; Informed consent; Deception

\section{Introduction}

Placebos may be used in the clinical trial context or in the clinical practice context. In the former case, particularly under single or double-blind conditions, if participants have given informed consent to possibly be allocated into the placebo arm, the issue of whether placebo controls fail to respect patients' right of autonomy does not arise because no deception is involved. However, given that placebo interventions are inert $^{1}$, they may violate the beneficent-based requirement of the original Helsinki Declaration, which requires that participants "should be assured of the best proven diagnostic and therapeutic method" [1]. In 2008, the Declaration was amended to allow the use of placebo when it "is necessary to determine the efficacy or safety of an intervention and the patients who receive placebo or no treatment will not be subject to any risk of serious or irreversible harm." But what is the rationale behind this amendment? In the clinical practice context, on the contrary, as placebos are usually used as a last resort, the issue of whether placebo use violates the beneficent-based requirement is not salient. But placebos are usually used deceptively and this practice may fail to respect patients' autonomy. In this paper, supporting the amendment of the Helsinki Declaration, I am arguing that placebo-controlled trials are ethically justified. Banning such trials will harm the prospective participants' interests as well as the end-users' of new treatments. In the clinical practice context, contrary to what some ethicists argue (e.g., [2]), I contend that the deceptive use of placebo may not violate patients' right of autonomy and thereby can also be ethically justified.

\section{Clinical trials}

I agree with the amendment of the Helsinki Declaration. When patients participating in a study give informed consent to receive an active or a placebo treatment, they are willing to receive a less-thanbest-proven intervention. Forcing them to receive the best proven intervention is similar to forcing someone to quit smoking or to stop skydiving; both acts are paternalistic. Paternalism is justified only when the patient's autonomy has already been or is going to be compromised. For example, transfusing blood to Jehovah's Witnesses against their will when the transfusion is necessary for their survival is morally justified given that one of their long-term values is to live according to the Bible. Their actions are non-autonomous because they are largely inconsistent with their long-term values. Nonetheless, when patients participate in a new treatment's experimental study, they take a risk of nonfatal and reversible harm in order to possibly benefit themselves as well as other end-users of the treatment. Such actions are consistent with the value of having a flourishing life; and are more rational and autonomous than smoking or skydiving, which involves risking a fatal harm in order to obtain pleasure or excitement $^{2}$. Therefore, if smokers or skydivers make autonomous decisions in choosing their habits or sports even though they have long life as their long-term values, so do the trial participants. Hence, the revision of the Helsinki Declaration is justified. Furthermore, according to Dunlop and Banja, regarding the patients of major depression and anxiety disorder, "the currently existing drugs help approximately one-third of patients achieve remission and another third to achieve a good response (that falls short of a full remission of symptoms)" [3]. Given that placebos have no side-effects, they are the best proven treatment for about one-third of these patients.

In addition, Dunlop and Banja have stated some conditions in which equivalency trials (comparing the effects of a new drug and those of an established drug) cannot replace placebo-controlled trials. For example, if both the study drug and the established drug have a side-effect that is also a symptom of the disease in question, the researcher does not know how much the new drug contributes to the symptom [3]. Banning placebo-controlled studies will deprive the potential end-users, which include the participants, of a better drug.

\section{Clinical practice}

In the clinical practice context, placebos, as a last resort, are usually used deceptively because openly using placebos without including deception or concealment may undermine their efficacy [4]. In fact, no clear evidence has been found that placebo treatments can produce clinically significant benefits, with the possible exception of placebo

1 In this paper, I do not address "impure" placebos, which contain active medication with respect to certain diseases that are not at issue. For example, antibiotics are used as placebos with respect to viral infections.

2 I am assuming that autonomy admits a matter of degree. 
for acupuncture (i.e., random superficial needling at non-acupuncture points) as a pain relief [5]. Also very few studies have investigated the effect of open placebo use [2]. But suppose that a placebo treatment is effective only when it is used deceptively. Would deception make the treatment unethical? In other words, is informed consent always required for an autonomous medical decision?

Some may support the deceptive placebo use by arguing that in everyday life people often make deceptive statements to be kind or to avoid hurting others' feelings. My response is that, first, these white lies usually do not form the basis on which the listener makes important decisions. Second, even if the listener makes decisions based on them, medical decisions are different from the decisions in other areas. As Clarke has pointed out, regarding areas outside medicine, "If someone is competent to make decisions to consent, then they are generally regarded as entitled to do so in any way in which they see fit" [6]. By contrast, informed consent is required for an autonomous consent in medicine in order to make sure that the decision reflects, or is authenticated by, the patient's long-term values.

However, I think there is an exception to this requirement. I have argued that paternalistic treatments cannot be justified for patients who are in no danger of losing their autonomy. Hence, deception based on paternalistic reasons cannot be justified for them. Nonetheless, if the physician has evidence, not only a belief, that patients would rather be deceived in order to have an effective treatment, and if a rational person would also have the same preference if he or she is in such patients' positions, to deceive these patients is to respect their autonomy, just as to follow Odysseus' command to tie him to the mast in order to prevent him from being lured by Siren is to respect his autonomy. Given that the placebo is used as a last resort, given that the open use is ineffective, and given that patients are debriefed at the end of treatments, willing to receive the deceptive placebo can be a rational and autonomous choice.

\section{Conclusion}

In the clinical trial context, the amended Declaration of Helsinki is consistent in allowing the use of placebos and requires that "the interest of the individual has to prevail over the interest of science or society." Patients can participate autonomously in placebo-controlled trials that do not subject participants to serious or irreversible harm. Furthermore, participants will also be potentially benefited as they are potential end-users of the new treatments. In the clinical practice context, although Thomas has provided strong evidence that clinicianpatient interactions can influence the effects of treatment [7], no clear evidence exists that placebo treatments are generally effective. This is one of the reasons that placebos are usually used as a last resort. In such cases, assuming that open placebo use is ineffective, placebos can be used deceptively without violating patients' right of autonomy.

\section{References}

1. World Medical Association (1964) World Medical Association Declaration of Helsinki.

2. Asai A, Kadooka Y (2013) Reexamination of the ethics of placebo use in clinical practice. Bioethics 27: 186-193.

3. Dunlop BW, Banja J (2009) A renewed, ethical defense of placebocontrolled trials of new treatments for major depression and anxiety disorders. J Med Ethics 35: 384-389.

4. Cheyne C (2005) Exploiting placebo effects for therapeutic benefit. Health Care Anal 13: 177-188.

5. Miller FG, Colloca L (2009) The legitimacy of placebo treatments in clinical practice: evidence and ethics. Am J Bioeth 9: 39-47.

6. Clarke S (2001) Informed Consent in Medicine in Comparison with Consent in Other Areas of Human Activity. Southern Journal of Philosophy 39: 169-187.

7. Thomas KB (1987) General practice consultations: is there any point in being positive? Br Med J (Clin Res Ed) 294: 1200-1202. 